\title{
Proposed experiment to test fundamentally binary theories
}

\author{
Matthias Kleinmann, ${ }^{1, *}$ Tamás Vértesi, ${ }^{2, \dagger}$ and Adán Cabello ${ }^{3, \ddagger}$ \\ ${ }^{1}$ Department of Theoretical Physics, University of the Basque Country UPV/EHU, P.O. Box 644, E-48080 Bilbao, Spain \\ ${ }^{2}$ Institute for Nuclear Research, Hungarian Academy of Sciences, H-4001 Debrecen, P.O. Box 51, Hungary \\ ${ }^{3}$ Departamento de Física Aplicada II, Universidad de Sevilla, E-41012 Sevilla, Spain
}

\begin{abstract}
Fundamentally binary theories are nonsignaling theories in which measurements of many outcomes are constructed by selecting from binary measurements. They constitute a sensible alternative to quantum theory and have never been directly falsified by any experiment. Here we show that fundamentally binary theories are experimentally testable with current technology. For that, we identify a feasible Bell-type experiment on pairs of entangled qutrits. In addition, we prove that, for any $n$, quantum $n$-ary correlations are not fundamentally $(n-1)$-ary. For that, we introduce a family of inequalities that hold for fundamentally $(n-1)$-ary theories but are violated by quantum $n$-ary correlations.
\end{abstract}

\section{INTRODUCTION}

Quantum theory (QT) is the most successful theory physicists have ever devised. Still, there is no agreement on which physical reasons force its formalism [1]. It is therefore important to test "close-to-quantum" alternatives, defined as those which are similar to QT in the sense that they have entangled states, incompatible measurements, violation of Bell inequalities, and no experiment has falsified them, and sensible in the sense that they are in some aspects simpler than QT. Examples of these alternatives are theories allowing for almost quantum correlations [2], theories in which measurements are fundamentally binary [3], and theories allowing for a higher degree of incompatibility between binary measurements [4].

Each of these alternatives identifies a particular feature of QT that we do not fully understand and, as a matter of fact, may or may not be satisfied by nature. For example, we still do not know which principle singles out the set of correlations in QT [5]. In contrast, the set of almost quantum correlations satisfies a list of reasonable principles and is simple to characterize [2]. Similarly, we do not know why in QT there are measurements that cannot be constructed by selecting from binary measurements [3]. However, constructing the set of measurements of the theory would be simpler if this would not be the case. Finally, we do not know why the degree of incompatibility of binary measurements in QT is bounded as it is, while there are theories that are not submitted to such a limitation [4].

Unfortunately, we do not yet have satisfactory answers to these questions. Therefore, it is important to test whether nature behaves as predicted by QT also in these particular aspects. However, this is not an easy task. Testing almost quantum theories is difficult because we still do not have a well-defined theory; thus, there is not

\footnotetext{
* matthias_kleinmann001@ehu.eus

$\dagger$ tvertesi@atomki.mta.hu

$\ddagger$ adan@us.es
}

a clear indication on how we should aim our experiments. Another reason, shared by theories with larger binary incompatibility, is that the only way to test them is by proving that QT is wrong, which is, arguably, very unlikely. The case of fundamentally binary theories is different. We have explicit theories [3] and we know that fundamentally binary theories predict supraquantum correlations for some experiments but subquantum correlations for others. That is, if QT is correct, there are experiments that can falsify fundamentally binary theories [3]. The problem is that all known cases of subquantum correlations require visibilities that escape the scope of current experiments.

This is particularly unfortunate now that, after years of efforts, we have loophole-free Bell inequality tests [6$10]$, tests touching the limits of QT $[11,12]$, and increasingly sophisticated experiments using high-dimensional two-photon entanglement [13-15]. Therefore, a fundamental challenge is to identify a feasible experiment questioning QT beyond the local realistic theories [16].

The main aim of this work is to present a feasible experiment capable of excluding fundamentally binary theories. In addition, the techniques employed to identify that singular experiment will allow us to answer a question raised in Ref. [3], namely, whether or not, for some $n$, quantum $n$-ary correlations are fundamentally $(n-1)$ ary.

\section{A. Device-independent scenario}

Consider a bipartite scenario where two observers, Alice and Bob, perform independent measurements on a joint physical system. For a fixed choice of measurements $x$ for Alice and $y$ for Bob, $P(a, b \mid x, y)$ denotes the joint probability of Alice obtaining outcome $a$ and Bob obtaining outcome $b$. We assume that both parties act independently in the sense that the marginal probability for Alice to obtain outcome $a$ does not depend on the choice of Bob's measurement $y$, i.e., $\sum_{b} P(a, b \mid x, y) \equiv P\left(a,_{-} \mid x,_{-}\right)$, and analogously $\sum_{a} P(a, b \mid x, y) \equiv P\left({ }_{-},\left.b\right|_{-}, y\right)$. These are the nonsignaling conditions, which are obeyed by QT 
whenever both observers act independently, in particular, if the operations of the observers are spacelike separated. However, QT does not exhaust all possible correlations subject to these constraints [17].

The strength of this scenario lies in the fact that the correlations can be obtained without taking into account the details of the experimental implementation and hence it is possible to make statements that are independent of the devices used. This device-independence allows us to test nature without assuming a particular theory - such as QT - for describing any of the properties of the measurement setup. This way, it is also possible to make theory-independent statements and, in particular, to analyze the structure of any probabilistic theory that obeys the nonsignaling conditions.

\section{B. Fundamentally binary theories}

One key element of the structure of any probabilistic theory was identified in Ref. [3] and concerns how the set of measurements is constructed, depending on the number of outcomes. According to Ref. [3], it is plausible to assume that a theory describing nature has, on a fundamental level, only measurements with two outcomes while situations where a measurement has more outcomes are achieved by classical postprocessing of one or several two-outcome measurements. To make this a consistent construction, it is also admissible that the classical postprocessing depends on additional classical information and, in the bipartite scenario, this classical information might be correlated between both parties. The total correlation attainable in such a scenario are the binary nonsignaling correlations, which are characterized by the convex hull of all nonsignaling correlations obeying $P\left(a,,_{-} \mid x,_{-}\right)=0$ for all measurements $x$ and all but two outcomes $a$, and $P\left({ }_{-},\left.b\right|_{-}, y\right)=0$ for all measurements $y$ and all but two outcomes $b$. The generalization to $n$-ary nonsignaling correlations is straightforward.

In Ref. [3], it was shown that for no $n$ the set of $n$-ary nonlocal correlations covers all the set of quantum correlations. Albeit this being a general result, the proof in Ref. [3] has two drawbacks: (i) It does not provide a test which is experimentally feasible. (ii) It does not allow us to answer whether or not quantum $n$-ary correlations are still fundamentally $(n-1)$-ary. For example, the proof in Ref. [3] requires 10-outcome quantum measurements for excluding the binary case. In this work, we address both problems and provide (i') an inequality that holds for all binary nonsignaling correlations, but can be violated using three-level quantum systems (qutrits) with current technology, and (ii') a family of inequalities obeyed by $(n-1)$-ary nonsignaling correlations but violated by quantum measurements with $n$ outcomes.

\section{RESULTS}

\section{A. Feasible experiment to test fundamentally binary theories}

We first consider the case where Alice and Bob both can choose between two measurements, $x=0,1$ and $y=$ 0,1 , and each measurement has three outcomes $a, b=$ $0,1,2$. For a set of correlations $P(a, b \mid x, y)$, we define

$$
I_{a}=\sum_{k, x, y=0,1}(-1)^{k+x+y} P(k, k \mid x, y),
$$

where the outcomes with $k=2$ do not explicitly appear. With the methods explained in Sec. III A, we find that, up to relabeling of the outcomes,

$$
I_{a} \leq 1
$$

holds for nonsignaling correlations if and only if the correlations are fundamentally binary. However, according to QT, the inequality in Eq. (2) is violated, and a value of

$$
I_{a}=2(2 / 3)^{3 / 2} \approx 1.0887
$$

can be achieved by preparing a two-qutrit system in the pure state

$$
|\psi\rangle=\frac{1}{2}(\sqrt{2}|00\rangle+|11\rangle-|22\rangle)
$$

and choosing the measurements $x, y=0$ as $M_{k \mid 0}=$ $V|k\rangle\langle k| V^{\dagger}$, and the measurements $x, y=1$ as $M_{k \mid 1}=$ $U|k\rangle\langle k| U^{\dagger}$, where, in canonical matrix representation,

$$
V=\frac{1}{\sqrt{12}}\left(\begin{array}{ccc}
2 & 2 & 2 \\
-\sqrt{3}-1 & \sqrt{3}-1 & 2 \\
\sqrt{3}-1 & -\sqrt{3}-1 & 2
\end{array}\right)
$$

and $U=\operatorname{diag}(-1,1,1) V$.

Using the second level of the Navascués-Pironio-Acín (NPA) hierarchy [18], we verify that the value in Eq. (3) is optimal within our numerical precision of $10^{-6}$. The visibility required to observe a violation of the inequality in Eq. (2) is $91.7 \%$, since the value for the maximally mixed state is $I_{a}=0$. The visibility is defined as the minimal $p$ required to obtain a violation assuming that the prepared state is a mixture of the target state and a completely mixed state, $\rho_{\text {prepared }}=p|\psi\rangle\langle\psi|+(1-p) \rho_{\text {mixed }}$.

We show in Sec. III A that the inequality in Eq. (2) holds already if only one of the measurements of either Alice or Bob is fundamentally binary. Therefore, the violation of the inequality in Eq. (2) allows us to make an even stronger statement, namely, that none of the measurements used is fundamentally binary, thus providing a device-independent certificate of the genuinely ternary character of all measurements in the experimental setup.

The conclusion at this point is that the violation of the inequality in Eq. (2) predicted by QT could be experimentally observable even achieving visibilities that have 
been already attained in previous Bell-inequality experiments on qutrit-qutrit systems [13-15]. It is important to point out that, in addition, a compelling experiment requires that the local measurements are implemented as measurements with three outcomes rather than measurements that are effectively two-outcome measurements. That is, there should be a detector in each of the three possible outcomes of each party. The beauty of the inequality in Eq. (2) and the simplicity of the required state and measurements suggest that this experiment could be carried out in the near future.

\section{B. Quantum n-ary correlations are not fundamentally $(n-1)$-ary}

If our purpose is to test whether or not one particular measurement is fundamentally binary (rather than all of them), then it is enough to consider a simpler scenario where Alice has a two-outcome measurement $x=0$ and a three-outcome measurement $x=1$, while Bob has three two-outcome measurements $y=0,1,2$. We show in Sec. III A that for the combination of correlations

$$
I_{b}=-P\left(0,,_{-} \mid 0,_{-}\right)+\sum_{k=0,1,2}[P(0,0 \mid 0, k)-P(k, 0 \mid 1, k)],
$$

up to relabeling of the outcomes and Bob's measurement settings,

$$
I_{b} \leq 1
$$

holds for nonsignaling correlations if and only if the correlations are fundamentally binary. According to QT, this bound can be violated with a value of

$$
I_{b}=\sqrt{16 / 15} \approx 1.0328
$$

by preparing the state

$$
|\psi\rangle=\frac{1}{\sqrt{(3 \zeta+1)^{2}+2}}(|00\rangle+|11\rangle+|22\rangle+\zeta|\phi\rangle|\phi\rangle),
$$

where $\zeta=-\frac{1}{3}+\frac{1}{6} \sqrt{10 \sqrt{15}-38} \approx-0.19095,|\phi\rangle=|0\rangle+$ $|1\rangle+|2\rangle$, and choosing Alice's measurement $x=0$ as $A_{0 \mid 0}=\mathbb{1}-A_{1 \mid 0}, A_{1 \mid 0}=|\phi\rangle\langle\phi| / 3$, and measurement $x=1$ as $A_{k \mid 1}=|k\rangle\langle k|$, for $k=0,1,2$, and Bob's measurements $y=0,1,2$ as $B_{0 \mid y}=\mathbb{1}-B_{1 \mid y}$ and $B_{1 \mid k}=\left|\eta_{k}\right\rangle\left\langle\eta_{k}\right| /\left\langle\eta_{k} \mid \eta_{k}\right\rangle$, where $\left|\eta_{k}\right\rangle=|k\rangle+\xi|\phi\rangle$, for $k=0,1,2$, and $\xi=-\frac{1}{3}+$ $\frac{1}{6} \sqrt{6 \sqrt{15}+22} \approx 0.78765$. [Another optimal solution is obtained by flipping the sign before the $\left(\frac{1}{6} \sqrt{ }\right)$-terms in $\xi$ and $\zeta$, yielding $\xi \approx-1.4543$ and $\zeta \approx-0.47572$.]

We use the third level of the NPA hierarchy to confirm that, within our numerical precision of $10^{-6}$, the value in Eq. (8) is optimal. Notice, however, that the visibility required to observe a violation of the inequality in Eq. (7) is $96.9 \%$. This contrasts with the $91.7 \%$ required for the inequality in Eq. (2) and shows how a larger number of outcomes allows us to certify more properties with a smaller visibility.

Nevertheless, what is interesting about the inequality in Eq. (7) is that it is a member of a family of inequalities and this family allows us to prove that, for any $n$, quantum $n$-ary correlations are not fundamentally $(n-1)$-ary, a problem left open in Ref. [3]. For that, we modify the scenario used for the inequality in Eq. (7), so that now Alice's measurement $x=1$ has $n$ outcomes, while Bob has $n$ measurements with two outcomes. We let $I_{b}^{(n)}$ be as $I_{b}$ defined in Eq. (6), with the only modification that in the sum, $k$ takes values from 0 to $n-1$. Then,

$$
I_{b}^{(n)} \leq n-2
$$

is satisfied for all fundamentally $(n-1)$-ary correlations. The proof is given in Sec. III B. Clearly, the value $I_{b}^{(n)}=$ $n-2$ can already be reached by choosing the fixed local assignments where all measurements of Alice and Bob always have outcome $a, b=0$. According to QT, it is possible to reach values of $I_{b}^{(n)}>(n-2)+1 /\left(4 n^{3}\right)$, as can be found by generalizing the quantum construction from above to $n$-dimensional quantum systems with $\xi=\sqrt{2}$ and $\zeta=-1 / n+1 /\left(\sqrt{2} n^{2}\right)$. Thus, the $(n-1)$-ary bound is violated already by $n$-ary quantum correlations. Note, that the maximal quantum violation is already very small for $n=4$ as the bound from the third level of the NPA hierarchy is $I_{b}^{(4)}<2.00959$.

\section{METHODS}

\section{A. Restricted nonsignaling polytopes}

We now detail the systematic method that allows us to obtain the inequalities in Eqs. (2), (7), and (10). We write $S=\left[a_{1}, a_{2}, \ldots, a_{n}: b_{1}, b_{2}, \ldots, b_{m}\right]$ for the case where Alice has $n$ measurements and the first measurement has $a_{1}$ outcomes, the second $a_{2}$ outcomes, etc., and similarly for Bob and his $m$ measurements with $b_{1}, b_{2}, \ldots$, outcomes. The nonsignaling correlations for such a scenario form a polytope $C(S)$. For another bipartite scenario $S^{\prime}$ we consider all correlations $P^{\prime} \in C\left(S^{\prime}\right)$ that can be obtained by local classical postprocessing from any $P \in C(S)$. The convex hull of these correlations is again a polytope and is denoted by $C\left(S \rightarrow S^{\prime}\right)$.

The simplest nontrivial polytope of fundamentally binary correlations is then $C([2,2: 2,2] \rightarrow[3,3: 3,3])$. We construct the vertices of this polytope and compute the 468 facet inequalities (i.e., tight inequalities for fundamentally binary correlations) with the help of the Fourier-Motzkin elimination implemented in the software porta [19]. We confirm the results by using the independent software ppl [20]. Up to relabeling of the outcomes, only the facet $I_{a} \leq 1$ is not a face of the set the nonsignaling correlations $C([3,3: 3,3])$, which concludes our construction of $I_{a}$. In addition, we find that

$$
C([2,3: 3,3])=C([2,2: 2,2] \rightarrow[2,3: 3,3]),
$$


and therefore the inequality in Eq. (2) holds for all nonsignaling correlations where at least one of the measurements is fundamentally binary.

As a complementary question we consider the case where only a single measurement has three outcomes. According to Eq. (11), the smallest scenarios where such a verification is possible are $[2,3: 2,2,2]$ and $[2,2: 2,2,3]$. We first find that $C([2,2: 3,3,3])=C([2,2: 2,2,2] \rightarrow$ $[2,2: 3,3,3])$, i.e., even if all of Bob's measurements would be fundamentally ternary, the correlations are always within the set of fundamentally binary correlations. Hence, we investigate the polytope $C([2,2: 2,2,2] \rightarrow$ $[2,3: 2,2,2])$ and its 126 facets. Up to symmetries, only the facet $I_{b} \leq 1$ is not a face of $C([2,3: 2,2,2])$.

Our method also covers other scenarios. As an example we study the polytope $C([2,4: 2,4] \rightarrow[2,2,2: 2,2,2])$ with its 14052 facets. In this case, the four-outcome measurements have to be distributed to two-outcome measurements (or the two-outcome measurement is used twice). Hence, this scenario is equivalent to the requirement that for each party at least two of the three measurements are compatible. The polytope has, up to relabeling, 10 facets that are not a face of $C([2,2,2: 2,2,2])$. According to the fourth level of the NPA hierarchy, two of the facets may intersect with the quantum correlations. While for one of them the required visibility (with respect to correlations where all outcomes are equally probable) is at least $99.94 \%$, the other requires a visibility of at least $97.88 \%$. This latter facet is $I_{c} \leq 0$, where

$$
\begin{gathered}
I_{c}=-P(10 \mid 00)-P(00 \mid 01)-P(00 \mid 10)-P(00 \mid 11) \\
-P(10 \mid 12)-P(01 \mid 20)-P(01 \mid 21)+P(00 \mid 22) .
\end{gathered}
$$

For arbitrary nonsignaling correlations, $I_{c} \leq 1 / 2$ is tight, while within QT, $I_{c}<0.0324$ must hold. We can construct a numeric solution for two qutrits which matches the bound from the third level of the NPA hierarchy up to our numerical precision of $10^{-6}$. The required quantum visibility then computes to $97.2 \%$. The quantum optimum is reached for measurements $A_{0 \mid k}=\left|\alpha_{k}\right\rangle\left\langle\alpha_{k}\right|$, $A_{1 \mid k}=\mathbb{1}-A_{0 \mid k}$, and $B_{0 \mid k}=\left|\beta_{k}\right\rangle\left\langle\beta_{k}\right|, B_{1 \mid k}=\mathbb{1}-B_{0 \mid k}$, where all $\left|\alpha_{k}\right\rangle$ and $\left|\beta_{k}\right\rangle$ are normalized and $\left\langle\alpha_{0} \mid \alpha_{1}\right\rangle \approx$ $0.098,\left\langle\alpha_{0} \mid \alpha_{2}\right\rangle \approx 0.630,\left\langle\alpha_{1} \mid \alpha_{2}\right\rangle \approx 0.572$, and $\left\langle\beta_{k} \mid \beta_{\ell}\right\rangle \approx$ 0.771 for $k \neq \ell$. A state achieving the maximal quantum value is $|\psi\rangle \approx 0.67931|00\rangle+0.67605|11\rangle+0.28548|22\rangle$. Note, that $I_{c} \approx 0.0318$ can still be reached according to QT, when Alice has only two incompatible measurements by choosing $\left\langle\alpha_{0} \mid \alpha_{1}\right\rangle=0$. Curiously, the facet $I_{c} \leq 0$ is equal to the inequality $M_{3322}$ in Ref. [21] and a violation of it has been observed recently by using photonic qubits [12]. However, while $M_{3322}$ is the only nontrivial facet of the polytope investigated in Ref. [21], it is just one of several nontrivial facets in our case.

\section{B. Proof of the inequality in Eq. (10)}

Here, we show that for $(n-1)$-ary nonsignaling correlations, the inequality in Eq. (10) holds. We start by letting for some fixed index $0 \leq \ell<n$,

$$
\begin{aligned}
F & =-\sum_{b} R_{0, b \mid 0, \ell}+\sum_{k}\left[R_{0,0 \mid 0, k}-R_{k, 0 \mid 1, k}\right], \\
X_{1 ; a \mid x, y} & =\sum_{b}\left(R_{a, b \mid x, y}-R_{a, b \mid x, \ell}\right) \\
X_{2 ; b \mid x, y} & =\sum_{a}\left(R_{a, b \mid x, y}-R_{a, b \mid 0, y}\right),
\end{aligned}
$$

where all $R_{a, b \mid x, y}$ are linearly independent vectors from a real vector space $V$. Clearly, for any set of correlations, we can find a linear function $\phi: V \rightarrow \mathbb{R}$ with $\phi\left(R_{a, b \mid x, y}\right)=$ $P(a, b \mid x, y)$. For such a function, $I_{b}^{(n)}=\phi(F)$ holds and $\phi\left(X_{\tau}\right)=0$ are all the nonsignaling conditions. The maximal value of $I_{b}^{(n)}$ for $(n-1)$-ary nonsignaling correlations is therefore given by

$$
\begin{aligned}
\max _{\ell^{\prime}} \max \{\phi(F) \mid & \phi: V \rightarrow \mathbb{R}, \text { linear, } \\
& \phi\left(X_{\tau}\right)=0, \text { for all } \tau, \\
& \phi\left(R_{\ell^{\prime}, b \mid 1, y}\right)=0, \text { for all } b, y, \\
& \sum_{v} \phi\left(R_{v}\right)=2 n, \text { and } \\
& \left.\phi\left(R_{v}\right) \geq 0, \text { for all } v\right\} .
\end{aligned}
$$

Since the value of the inner maximization does not depend on the choice of $\ell$, we can choose $\ell=\ell^{\prime}$. Equation (14) is a linear program, and the equivalent dual to this program can be written as

$$
\max _{\ell} \min _{t, \boldsymbol{\xi}, \boldsymbol{\eta}}\left\{t \mid t \geq \zeta_{v} \text { for all } v\right\},
$$

where $\boldsymbol{\zeta}$ is the solution of

$$
2 n F-\sum_{\tau} \xi_{\tau} X_{\tau}-\sum_{b, y} \eta_{b, y} R_{\ell, b \mid 1, y}=\sum_{v} \zeta_{v} R_{v}
$$

To obtain an upper bound in Eq. (15), we choose $\boldsymbol{\eta} \equiv 2 n$ and all $\xi_{\tau}=0$, but $\xi_{1 ; a \mid 0, k}=4, \xi_{1 ; k \mid 1, k}=-2 n, \xi_{2 ; b \mid 1, \ell}=$ $-3 n+2$, and $\xi_{2 ; b \mid 1, k}=-(-1)^{b} n+2$, for $k \neq \ell$. This yields $\max _{v} \zeta_{v}=n-2$ for all $\ell$ and hence the $(n-1)$-ary nonsignaling correlations obey $I_{b}^{(n)} \leq n-2$.

\section{CONCLUSIONS}

There was little chance to learn new physics from the recent loophole-free experiments of the Bell inequality [610]. Years of convincing experiments [22-24] allowed us to anticipate the conclusions: nature cannot be explained by local realistic theories [16], there are measurements for which there is not a joint probability distribution [25], and there are states that are not a convex combination of local states [26].

Here we have shown how to use Bell-type experiments to gain insights into QT. In Ref. [3], it was shown that QT predicts correlations that cannot be explained by nonsignaling correlations produced by fundamentally binary measurements (including Popescu-Rohrlich boxes 
[17]). We proposed a feasible experiment which will allow us to either exclude all fundamentally binary probabilistic theories or to falsify QT. If the results of the experiment violate the inequality in Eq. (2), as predicted by QT, then we would learn that no fundamentally binary theory can possibly describe nature. In addition, it would prove that all involved measurements are genuine three-outcome measurements. If the inequality in Eq. (2) is not violated despite visibilities would a priori lead to such a violation, then we would have evidence that QT is wrong at a fundamental level (although being subtle to detect in experiments). We have also gone beyond Ref. [3] by showing that, for any $n$, already $n$-ary

[1] G. Chiribella and R. W. Spekkens (eds.), Quantum Theory: Informational Foundations and Foils, Fundamental Theories of Physics, Vol. 181 (Springer, Dordrecht, Holland, 2016).

[2] M. Navascués, Y. Guryanova, M. J. Hoban, and A. Acín, Almost quantum correlations, Nat. Comm. 6, 6288 (2015).

[3] M. Kleinmann and A. Cabello, Quantum Correlations Are Stronger Than All Nonsignaling Correlations Produced by $n$-Outcome Measurements, Phys. Rev. Lett. 117, 150401 (2016).

[4] P. Busch, T. Heinosaari, J. Schultz, and N. Stevens, Comparing the degrees of incompatibility inherent in probabilistic physical theories, EPL 103, 10002 (2013).

[5] A. Cabello, Simple Explanation of the Quantum Limits of Genuine $n$-Body Nonlocality, Phys. Rev. Lett. 114, 220402 (2015).

[6] B. Hensen, H. Bernien, A. E. Dréau, A. Reiserer, N. Kalb, M. S. Blok, J. Ruitenberg, R. F. L. Vermeulen, R. N. Schouten, C. Abellán, W. Amaya, V. Pruneri, M. W. Mitchell, M. Markham, D. J. Twitchen, D. Elkouss, S. Wehner, T. H. Taminiau, and R. Hanson, Loophole-free Bell inequality violation using electron spins separated by 1.3 kilometres, Nature (London) 526, 682 (2015).

[7] M. Giustina, M. A. M. Versteegh, S. Wengerowsky, J. Handsteiner, A. Hochrainer, K. Phelan, F. Steinlechner, J. Kofler, J.-A. Larsson, C. Abellán, W. Amaya, V. Pruneri, M. W. Mitchell, J. Beyer, T. Gerrits, A. E. Lita, L. K. Shalm, S. W. Nam, T. Scheidl, R. Ursin, B. Wittmann, and A. Zeilinger, Significant-LoopholeFree Test of Bell's Theorem with Entangled Photons, Phys. Rev. Lett. 115, 250401 (2015).

[8] L. K. Shalm, E. Meyer-Scott, B. G. Christensen, P. Bierhorst, M. A. Wayne, M. J. Stevens, T. Gerrits, S. Glancy, D. R. Hamel, M. S. Allman, K. J. Coakley, S. D. Dyer, C. Hodge, A. E. Lita, V. B. Verma, C. Lambrocco, E. Tortorici, A. L. Migdall, Y. Zhang, D. R. Kumor, W. H. Farr, F. Marsili, M. D. Shaw, J. A. Stern, C. Abellán, W. Amaya, V. Pruneri, T. Jennewein, M. W. Mitchell, P. G. Kwiat, J. C. Bienfang, R. P. Mirin, E. Knill, and S. W. Nam, Strong Loophole-Free Test of Local Realism, Phys. Rev. Lett. 115, 250402 (2015).

[9] B. Hensen, N. Kalb, M. S. Blok, A. E. Dréau, A. Reis- quantum correlations are not fundamentally $(n-1)$-ary.

\section{ACKNOWLEDGMENTS}

This work is supported by Project No. FIS2014-60843P, "Advanced Quantum Information" (MINECO, Spain), with FEDER funds, the FQXi Large Grant "The Observer Observed: A Bayesian Route to the Reconstruction of Quantum Theory", the project "Photonic Quantum Information" (Knut and Alice Wallenberg Foundation, Sweden), the Hungarian National Research Fund OTKA (Grants No. K111734 and No. KH125096), the EU (ERC Starting Grant GEDENTQOPT), and the DFG (Forschungsstipendium KL 2726/2-1). erer, R. F. L. Vermeulen, R. N. Schouten, M. Markham, D. J. Twitchen, K. Goodenough, D. Elkouss, S. Wehner, T. H. Taminiau, and R. Hanson, Loophole-free Bell test using electron spins in diamond: Second experiment and additional analysis Sci. Rep. 6, 30289 (2016).

[10] W. Rosenfeld, D. Burchardt, R. Garthoff, K. Redeker, N. Ortegel, M. Rau, and H. Weinfurter, Event-Ready Bell Test Using Entangled Atoms Simultaneously Closing Detection and Locality Loopholes Phys. Rev. Lett. 119, 010402 (2017).

[11] H. S. Poh, S. K. Joshi, A. Cerè, A. Cabello, and C. Kurtsiefer, Approaching Tsirelson's Bound in a Photon Pair Experiment, Phys. Rev. Lett. 115, 180408 (2015).

[12] B. G. Christensen, Y.-C. Liang, N. Brunner, N. Gisin, and $\mathrm{P}$.G. Kwiat, Exploring the Limits of Quantum Nonlocality with Entangled Photons, Phys. Rev. X 5, 041052 (2015).

[13] A. Vaziri, G. Weihs, and A. Zeilinger, Experimental TwoPhoton, Three-Dimensional Entanglement for Quantum Communication, Phys. Rev. Lett. 89, 240401 (2002).

[14] S. Gröblacher, T. Jennewein, A. Vaziri, G. Weihs, and A. Zeilinger, Experimental quantum cryptography with qutrits, New. J. Phys. 8, 75 (2006).

[15] A. C. Dada, J. Leach, G. S. Buller, M. J. Padgett, and E. Andersson, Experimental high-dimensional two-photon entanglement and violations of generalized Bell inequalities, Nat. Phys. 7, 677 (2011).

[16] J. S. Bell, On the Einstein Podolsky Rosen paradox, Physics (Long Island City, NY) 1, 195 (1964).

[17] S. Popescu and D. Rohrlich, Quantum nonlocality as an axiom, Found. Phys. 24, 379 (1994).

[18] M. Navascués, S. Pironio, and A. Acín, Bounding the Set of Quantum Correlations, Phys. Rev. Lett. 98, 010401 (2007).

[19] POlyhedron Representation Transformation Algorithm, retrieved from http://porta.zib.de/.

[20] Parma Polyhedra Library, retrieved from http://bugseng.com/products/ppl/.

[21] N. Brunner, N. Gisin, and V. Scarani, Entanglement and non-locality are different resources, New. J. Phys. 7, 88 (2005).

[22] S. J. Freedman and J. F. Clauser, Experimental Test of Local Hidden-Variable Theories, 
Phys. Rev. Lett. 28, 938 (1972).

[23] A. Aspect, J. Dalibard, and G. Roger, Experimental Test of Bell's Inequalities Using Time-Varying Analyzers, Phys. Rev. Lett. 49, 1804 (1982).

[24] G. Weihs, T. Jennewein, C. Simon, H. Weinfurter, and A. Zeilinger, Violation of Bell's In- equality under Strict Einstein Locality Conditions, Phys. Rev. Lett. 81, 5039 (1998).

[25] A. Fine, Hidden Variables, Joint Probability, and the Bell Inequalities, Phys. Rev. Lett. 48, 291 (1982).

[26] R. F. Werner, Quantum states with Einstein-PodolskyRosen correlations admitting a hidden-variable model, Phys. Rev. A 40, 4277 (1989). 